EUROPEAN ORGANIZATION FOR NUCLEAR RESEARCH

European Laboratory for Particle Physics

Large Hadron Collider Project

LHC Project Report 505

\title{
HE II HEAT EXCHANGER TEST UNIT FOR THE LHC INNER TRIPLET
}

\author{
Ch. Darve', E. Blanco ${ }^{2}$, Y. Huang ${ }^{1}$, T. Nicol', \\ T. Peterson ${ }^{1}$ and R. van Weelderen ${ }^{2}$
}

\begin{abstract}
The Inner Triplet Heat Exchanger Test Unit (IT-HXTU) is a 30-m long thermal model designed at Fermilab, built in US industry, fully automated and tested at CERN as part of the US LHC program to develop the LHC Interaction Region quadrupole system. The cooling scheme of the IT-HXTU is based on heat exchange between stagnant pressurized He II in the magnet cold mass and saturated He II (two-phase) flowing in a heat exchanger located outside of and parallel to the cold mass.

The purposes of this test are, among others, to validate the proposed cooling scheme and to define an optimal control strategy to be implemented in the future LHC accelerator. This paper discusses the results for the heat exchanger test runs and emphasizes the thermal and hydraulic behavior of He II for the inner triplet cooling scheme.
\end{abstract}

1 Fermi National Accelerator Laboratory, Batavia, IL, 60510, USA. Member of the CERN-US Collaboration to the LHC Project

2 CERN, LHC Division

Presented at the 2001 Cryogenic Engineering Conference and International Cryogenic Materials Conference CEC/ICMC 2001

16-20 July 2001, Madison, Wisconsin, USA

Administrative Secretariat

LHC Division

CERN

CH - 1211 Geneva 23

Switzerland

Geneva, 19 October 2001 


\title{
He II HEAT EXCHANGER TEST UNIT FOR THE LHC INNER TRIPLET
}

\author{
Ch. Darve ${ }^{1}$, E. Blanco ${ }^{2}$, Y. Huang ${ }^{1}$, T. Nicol ${ }^{1}$, T. Peterson ${ }^{1}$ and Rob van \\ Weelderen ${ }^{2}$ \\ ${ }^{1}$ Fermi National Accelerator Laboratory, Batavia, IL, 60510, USA \\ ${ }^{2}$ CERN, European Organization for Particle Physics, Geneva, 1211, CH
}

\begin{abstract}
The Inner Triplet Heat Exchanger Test Unit (IT-HXTU) is a 30-m long thermal model designed at Fermilab, built in US industry, fully automated and tested at CERN as part of the US LHC program to develop the LHC Interaction Region quadrupole system. The cooling scheme of the IT-HXTU is based on heat exchange between stagnant pressurized He II in the magnet cold mass and saturated He II (two-phase) flowing in a heat exchanger located outside of and parallel to the cold mass.

The purposes of this test are, among others, to validate the proposed cooling scheme and to define an optimal control strategy to be implemented in the future LHC accelerator. This paper discusses the results for the heat exchanger test runs and emphasizes the thermal and hydraulic behavior of He II for the inner triplet cooling scheme.
\end{abstract}

\section{INTRODUCTION}

The $27 \mathrm{~km}$ circumference Large Hadron Collider (LHC) under construction at CERN will study the collision of protons at an energy at the center of mass up to $2 \times 7 \mathrm{TeV}$ and at a nominal luminosity of $10^{34} \mathrm{~cm}^{-2} \mathrm{~s}^{-1}$. Superconducting quadrupoles arranged in assemblies called "inner triplets" will focus the particle beam at each of the four interaction regions (IRs). One inner triplet is located on each side of each of the four IRs, resulting in eight inner triplets in the LHC. Each 30 meter long LHC inner triplet is composed of three cryostats, containing a total of four superconducting quadrupole magnets operating at $1.9 \mathrm{~K}$. In the inner triplet regions, beam-induced heating is of great concern. At nominal luminosity the heat load to $1.9 \mathrm{~K}$ will total about $200 \mathrm{~W}$ over $30 \mathrm{~m}$, compared to $40 \mathrm{~W}$ over the $107 \mathrm{~m}$ long arc magnet cells. To extract the large heat load of the IR, a 97.5-mm outer diameter corrugated copper heat exchanger tube is used, rather than a smooth 58-mm outer diameter for 2.3-mm wall thickness like for the arc magnets [1]. Due to the large tube 
diameter, the heat exchanger can only be located outside of and parallel to the magnet cold mass. Heat from the magnet is transported by conduction in stagnant, pressurized He II to the heat exchanger tube via a total of five connections at the magnet ends and interconnects where it is carried away by a flow of saturated two-phase superfluid helium.

The Inner Triplet Heat Exchanger Test Unit (IT-HXTU) is a full-scale thermal model of the inner triplet. It was designed at Fermilab and built by US industry as part of the US LHC Accelerator Collaboration program to develop the LHC IR quadrupole system [2-3]. It was fully automated and tested at CERN. The cooling scheme of the IT-HXTU is equivalent to the LHC IR inner triplet one. The LHC thermal load conditions were simulated with the use of the electrical heaters installed in the cold masses.

\section{PURPOSE OF THE TEST}

The IT-HXTU is a thermal model built for the purpose of measuring the LHC IR heat transfer from the beam-induced heating to the refrigeration system. The thermal model provides important data in the validation of the inner triplet design. The main purpose of this study is to validate the inner triplet cooling scheme by checking the temperature rise in the stagnant and pressurized He II due to the beam-induced heating in the inner triplet magnets. The temperature profile within the HXTU is measured for different simulated LHC thermal conditions. The thermal design requirement for the external heat exchanger is to remove nominally $200 \mathrm{~W}$ at $1.9 \mathrm{~K}$ with a temperature drop between the saturated and the pressurized He II limited to $50 \mathrm{mK}$. Several LHC heat load scenarios were studied. Twophase flow pressure drop between the inlet and outlet of the saturated He II flow in the heat exchanger tube was measured as well as temperatures in the pressurized superfluid.

Another purpose of this study was to investigate and develop an optimal temperature regulation strategy compatible with the methodology to be implemented in the regular cells of the LHC accelerator: a Nonlinear Model-Based Predictive Control (NMPC) [4].

\section{THERMAL MEASUREMENTS UNDER INVESTIGATION}

\section{Environment}

The heat is transported from the magnet simulator heat source, in the pressurized He II bath, and through the pressurized He II to the copper heat exchanger. Then the heat is transferred through the copper tube wall to the flowing two-phase saturated He II inside the tube. Along this path, two main heat transport principles are to be taken into account and measured. The first heat transport mechanism is the Gorter-Mellink heat transport through pressurized superfluid, and the second is the Kapitza effect at the heat exchanger wall. The conduction in pressurized superfluid helium and the Kapitza effect in between the solid-liquid interface with the He II and heat exchanger tube were both modeled and investigated in order to interpret the experimental results.

\section{Heat transfer in superfluid helium}

We analyzed the heat transfer in the HXTU to compare predicted temperatures with measurements. The one dimensional turbulent heat transport in a channel containing He II is expressed by the Gorter-Mellink equation: 


$$
\frac{d T}{d x}=-f(T) q^{m}
$$

where $\mathrm{q}$ is the heat flux in $\mathrm{W} / \mathrm{cm}^{2}$. The best fit for exponent $\mathrm{m}$ varies from 3 to nearly 4 [5] for various superfluid conditions, with some authors choosing $m=3.4$ as the best-fit [6]. For simplicity, we use $m=3$ in our calculations. The quantity $1 / f(T)$ is the effective thermal conductivity of $\mathrm{He}$ II and from $1.85 \mathrm{~K}$ to $1.95 \mathrm{~K}$ at 1 bar can be considered as a constant, $1200 \mathrm{~W}^{3} / \mathrm{cm}^{5} \mathrm{~K}$ for $\mathrm{m}=3$.

For the heat flux along the electric heaters and around the corrugated heat exchanger tube, delta-T's with a non-uniform heat flux were estimated. We assume the heat flux through the pressurized superfluid increases linearly with position (constant wall heat flux), and again assume $\frac{1}{f(T)}=$ const . One may then easily integrate $\frac{d T}{d x}=f(T) q^{m}$

over the length of the tube, where $q=q_{0}+\frac{Q x}{A}, \mathrm{q}_{0}$ is the heat flux through the superfluid into the system, $\mathrm{Q}$ is the heat added per unit length (e.g., W/m), $\mathrm{A}$ is the heat flow passage area, and $\mathrm{x}$ is distance along the heat flow passage. With $\mathrm{f}(\mathrm{T}), \mathrm{q}_{0}, \mathrm{Q}$, and $\mathrm{A}$ all constant, integrating from $\mathrm{x}=0$ to $\mathrm{x}=\mathrm{L}$ gives

$$
\Delta T=\frac{A}{(m+1) Q(1 / f(T))}\left\{\left(q_{0}+\frac{Q L}{A}\right)^{m+1}-q_{0}{ }^{m+1}\right\}
$$

Note that $q_{0}+\frac{Q L}{A}=q_{L}$ where $\mathrm{q}_{\mathrm{L}}$ is the heat flux at the $\mathrm{x}=\mathrm{L}$ end of the heat flow passage. Thus, the temperature difference through the passage may be written as

$$
\Delta T=\frac{A}{(m+1) Q(1 / f(T))}\left(q_{L}^{m+1}-q_{0}{ }^{m+1}\right)
$$

Equation (3) was used for estimating the temperature difference in regions with distributed heat input.

\section{Kapitza effect}

The temperature rise between the pressurized He II bath and the saturated $\mathrm{He}$ II flow is dominated by the Kapitza effect at the heat exchanger wall interface between the liquid and the solid. A preliminary test was performed at Fermilab in order to quantify this influence and to compare the performance of the current heat exchanger tube with different surface treatments of corrugated tubes. A short sample of the corrugated heat exchanger tube was tested under the LHC thermal conditions. The principle of this heat transport and the detailed results of this test are treated elsewhere [7]. The measured total thermal conductance yields a Kapitza coefficient of $893+/-3 \% \mathrm{~W} \cdot \mathrm{K}^{-4} \cdot \mathrm{m}^{-2}$. Therefore, a Kapitza conductance of $0.565 \mathrm{~W} \cdot \mathrm{K}^{-1} \cdot \mathrm{cm}^{-2}$ is estimated for the operating temperature of $1.85 \mathrm{~K}$. The contribution of the thermal conductivity of the tube to the impedance is negligible. Knowing the properties of the exchanger tube with the fact that this short sample of tube was wetted over $100 \%$ of its surface, we can determine the wetted area in each of the ITHXTU operation scenarios. 


\section{IT-HXTU TEST DESCRIPTION}

\section{Hardware, control system and instrumentation}

The 30-m long cooling scheme is composed of a feedbox, four identical modules and a turnaround box. Each module consists of a 143-mm outer diameter pipe instrumented with electric heaters and thermometers to simulate the cold mass and a 168-mm outer diameter upper pipe surrounding the corrugated copper pipe, serving as the heat exchanger. A vertical 89-mm outer diameter tube connects both pipes. The feed-box, the four modules and the turnaround are connected in series and inclined with a $1.4 \%$ slope to match the steepest inclination around the interaction regions in the LHC tunnel. A phase separator reservoir located in the feedbox is used to receive the liquid helium from the CERN test station. The vapor generated is then extracted from the top of the phase separator to cool the 30-m long thermal shield. The liquid helium is subcooled by cold helium vapor via a subcooling heat exchanger, and then expanded through a JT valve so that the saturated He II can be supplied at one side of the corrugated tube. The CERN cryogenic facility is capable of providing the mass flow-rate up to $18 \mathrm{~g} / \mathrm{s}$ at about $15 \mathrm{mbar}$. FIGURE 1 shows the Simplified Process and Instrumentation Diagram. A description of the instrumentation used is detailed in TABLE 1.

Due to the special architecture of the IT-HXTU setup, a distributed solution for the low-level communication has been adopted. An industrial Programmable Logical Controller (PLC) is located in the feedbox and connected to the sensors via a Profibus fieldbus and specialized I/O modules. A dedicated desktop PC placed in the control room, running PCVue32® as SCADA, is connected via an Ethernet network to acquire and supervise the equipment. Several closed control loops regulate levels, temperatures and pressures using classical PID controllers. For the $1.8 \mathrm{~K}$ Cooling Loop a new technique has been employed: a Nonlinear Model-based Predictive Controller (NMPC). This controller is running in a special PLC, an industrial PC running a real time operating system [4].

The temperature profiles within the pressurized He II are measured with Cernox ${ }^{\circledR}$ thermometers mounted on printed circuit boards (PCBs) at designated locations [8].

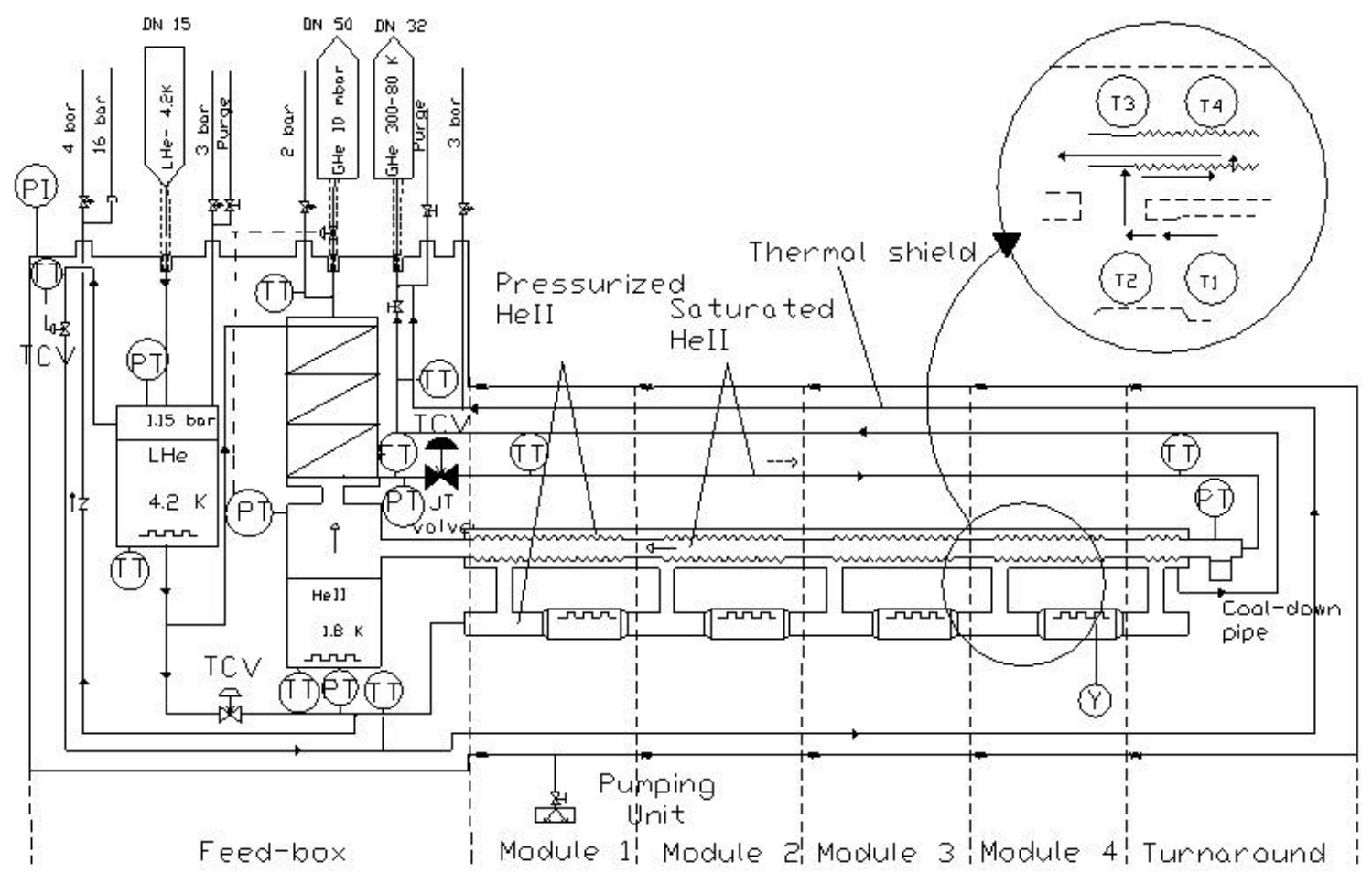

FIGURE 1. Simplified Process and Instrumentation Diagram 
TABLE 1. Inner Triplet mounted instrumentation

\begin{tabular}{lllll}
\hline Instrument & Total & Range & Accuracy & Syb. \\
\hline Temperature (Cernox®, Pt100) & 54 & $1.6-40 \mathrm{~K}, 50 \mathrm{~K}-300 \mathrm{~K}$ & $\pm 5 \mathrm{mK}, \pm 5 \mathrm{~K}$ & $\mathrm{TT}$ \\
Pressure (Absolute, Differential) & 5 & $0-1.3 \mathrm{bar}, 0-0.13 \mathrm{bar}, 0-7.5 \mathrm{mbar}$ & $0.2 \%, 0.03 \mathrm{mbar}$ & $\mathrm{PT}$ \\
Level (AMI) & 5 & $0-6 \%, 0-12 ”, 0-28$, & $\pm 2 \% \mathrm{FS}$ & $\mathrm{LT}$ \\
Flowmeter (Turbine) & 1 & $0-20 \mathrm{~g} / \mathrm{s}$ & $\pm 2 \% \mathrm{FS}$ & $\mathrm{FT}$ \\
Heaters (Electrical resistances) & 12 & $55,90,240 \mathrm{Watts}$ & $\mathrm{Y}$ \\
Control Valves & 6 & $0-100 \%$ & $\mathrm{TCV}$ \\
\hline
\end{tabular}

The vapor pressure drop along the saturated flowing He II is measured with both absolute pressure transducers located at the inlet and outlet of the corrugated heat exchanger tube and a differential pressure sensor. The saturated He II temperature (inside of the corrugated copper pipe) is determined by the vapor pressures at both ends of the heat exchanger.

\section{Measurement principle}

The first purpose is to study the temperature distribution in steady state mode while the environment of the heat exchanger corresponds to LHC thermal conditions. In order to analyze the heat exchanger performances and the heat transfer during the test, we controlled the following variables: The heat load, Q, generated by Joule-effect through electrical resistances to the pressurized He II, the saturated He II temperature and the massflow. The principle of the measurement is to fix the variables and to measure the temperature distribution corresponding to find the final thermal equilibrium conditions. The evolution of the dry out point toward the accumulator, in the heat exchanger tube, is the indicator of the thermal equilibrium. The measurements are taken for a controlled overflow of the saturated helium to the feed-box accumulator. The temperature differences measured between the pressurized and the saturated He II media are part of the results as well as the pressure drop in saturated He II along the heat exchanger tube.

\section{Commissioning}

During the installation of the test unit, the corrugated heat exchanger pipe deflection and sag of each independent module was checked. A dedicated tool was designed and manufactured for the purpose of running in the tube. 8-mm of maximum sag was measured.

The cool-down time was 2 days from room temperature to $4.2 \mathrm{~K}$ and half a day from $4.2 \mathrm{~K}$ to $1.9 \mathrm{~K}$. The velocity of the saturated He II, liquid phase was measured to be about $10 \mathrm{~cm} / \mathrm{s}$.

Once the system was cooled and stable at $1.9 \mathrm{~K}$, the re-calibration of all thermometers was needed to give us an accurate baseline for a temperature profile. The static heat load from the environment to the $1.9 \mathrm{~K}$ system was measured by considering the enthalpy balance of the system between the JT-valve and the accumulator. The static heat due to conduction and radiation from the ambient to the $1.9 \mathrm{~K}$ system is equal to $34 \mathrm{~W}$.

In addition, we tested the behavior of the heat exchanger test unit, by varying the location of the heat loads along the 4 modules. Unbalanced scenarios permitted us to check the response of the heat exchanger tube. 
For the helium at 1 bar and $2.5 \mathrm{~K}$ before the JT valve, and a $1.8 \mathrm{~K}$ saturated $\mathrm{He} \mathrm{II}$, the yield was $84 \%$ after expansion.

\section{RESULTS AND DISCUSSION}

Several scenarios of the LHC operation were simulated in addition to the determination of the heat exchanger performance.

FIGURE 2 shows the distribution of the temperature difference between the pressurized and saturated He II, T- Tsat, for the nominal LHC luminosity condition. In this configuration the heat distributed are equivalent to $65 \mathrm{~W}, 35 \mathrm{~W}, 58 \mathrm{~W}$ and $56 \mathrm{~W}$ respectively to the 4 magnet simulators Y1 (feed-box side) up to Y4 (turnaround side). The saturated temperature is equal to $1.852 \mathrm{~K}$. A mass flow of $12.7 \mathrm{~g} / \mathrm{s}$ is needed to extract the total heat of $248 \mathrm{~W}$. The temperature differences, T-Tsat, for this case are always lower than the $48.1 \mathrm{mK}$. The ultimate LHC luminosity condition was approached within the limit of the test unit. The saturated temperature was equivalent to $1.915 \mathrm{~K}$, (saturated $\mathrm{He}$ II pressure equivalent to $2.41 \mathrm{kPa}$ ). The sum of the heat loads equally distributed in the magnet simulator pipes was $316 \mathrm{~W}$, including $34 \mathrm{~W}$ of static loads. The total temperature difference between the pressurized and saturated He II, T- Tsat, is $72 \mathrm{mK}$ for this case. Hence, a heat load of 1.5 times nominal and a saturation temperature of $1.92 \mathrm{~K}$ still leaves the magnet just under $2.0 \mathrm{~K}$, a temperature at which tests have shown it can operate.

The wetted surface area of the corrugated pipe is determined by comparison with the test results obtained with the small-scale heat exchanger test [7] where $100 \%$ of the surface was wetted. The wetted surface area is about $22 \%$ on average.

In order to check the performance of the heat exchanger system over a variety of temperatures and heat loads, data are compared with analytical predictions in a spreadsheet modified from one developed for the inner triplet. As one can see in FIGURE 3, the agreement between prediction and data is very good. For DT5, across the heat exchanger wall, the agreement is forced by the use of an "effective wetted area" of $22 \%$ found in the IT-HXTU measurements. The slight difference between predicted and measured may be due to the difference of effective wetted area from the $22 \%$ assumed in the calculation. Still, DT5 dominates the total temperature difference. The other delta-T's are predicted from geometry, helium properties, and heat load. Some asymmetries exist between parallel heat flow paths to the heat exchanger. DT2, the delta-T through the interconnect pipe, is found assuming an arithmetic average of the lengths on the two sides $(48 \mathrm{~cm}+32 \mathrm{~cm}) / 2=$ $40 \mathrm{~cm}$ for the length of heat transport. The larger and more significant asymmetry is due to the ends of the HXTU assembly, where heat to the interconnects comes from only one side. This effect is ignored and delta-T's in the middle of the HXTU assembly is used.

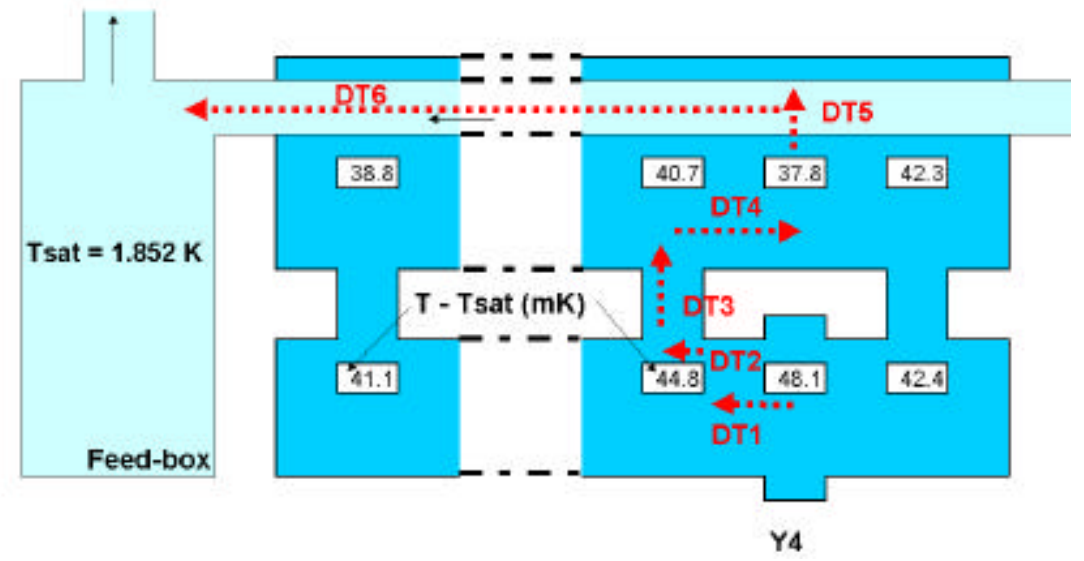

FIGURE 2. Distribution of the temperature for the LHC nominal luminosity conditions. 
DT1: from the Module 3 thermal center to the module end within the pressurized Helium II, linearly increasing heat flux

(length $=3.17 \mathrm{~m}$, diameter $=13.45 \mathrm{~cm}$ )

DT2: within the connecting pipe between modules within the pressurized Helium II, constant heat flux (length $=40 \mathrm{~cm}$, diameter $=8.28 \mathrm{~cm}$ )

DT3: between connecting pipe and heat exchanger within the pressurized Helium II, constant heat flux (length $=7.2 \mathrm{~cm}$, diameter $=8.28 \mathrm{~cm}$ )

DT4: within the pressurized Helium II side of the heat exchanger, linearly decreasing heat flux $\mathrm{L}=375 \mathrm{~cm}$, D_inner=9.6 cm, D_outer $=16 \mathrm{~cm}$.

DT5: across the He II heat exchanger wall

DT6: due to the vapor pressure drop.
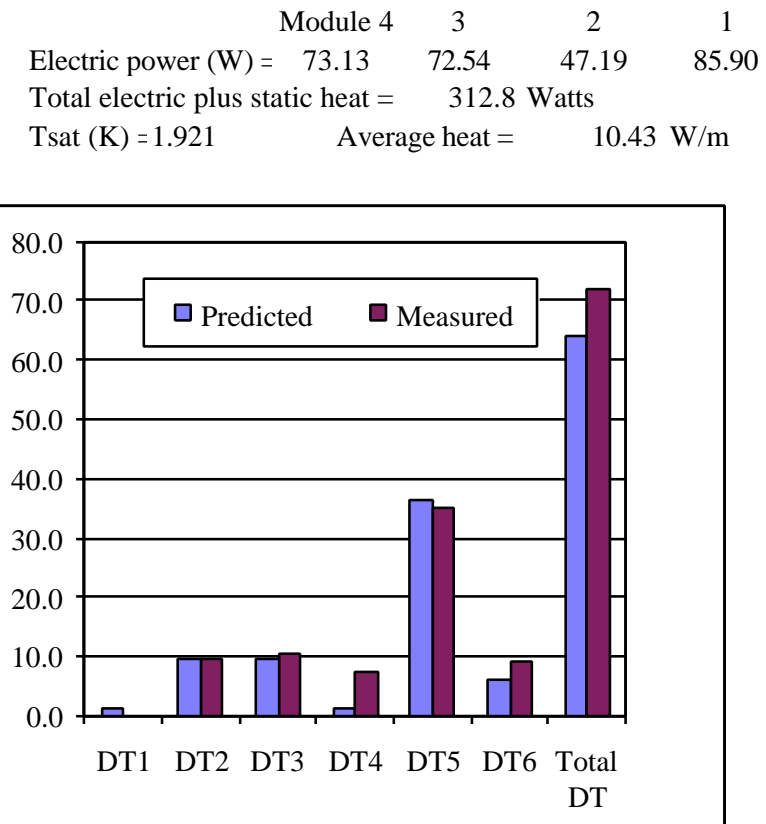

FIGURE 3. Comparison of measured and predicted delta-T's in the HXTU assembly for heating conditions and saturation conditions approximately 1.5 times the expected nominal heating in the inner triplets.

The measured pressure drop associated to the heat-input equal to $9.3 \mathrm{~W} / \mathrm{m}$ and $10.4 \mathrm{~W} / \mathrm{m}$ are $28 \mathrm{~Pa}$ and $73 \mathrm{~Pa}$, respectively. Hence the temperature drop DT6 is always lower than $10 \mathrm{mK}$ and is consistent with the calculation.

The biggest error in calculation compared to measurement is in DT4, the delta-T along the heat exchanger in pressurized He II. The error is generally of a few $\mathrm{mK}$, but it is a large percentage error, and is probably due to the effect of corrugated pipe supports (flow resistances) partially blocking heat flow along the heat exchanger. The DT4 discrepancy may also be due to the location of thermometers partly in the tee of the cross-connect rather than actually up in the heat exchanger pipe. The total temperature rise from saturated vapor to the simulated magnet cold mass is well within acceptable limits. With the nominal heat load and a saturation temperature of about $1.85 \mathrm{~K}$, which is expected in LHC, a temperature rise to the magnet of about $50 \mathrm{mK}$ puts the magnet at the design temperature of $1.90 \mathrm{~K}$. FIGURE 3 above shows that there is a good amount of margin in the heat transfer capability of the system.

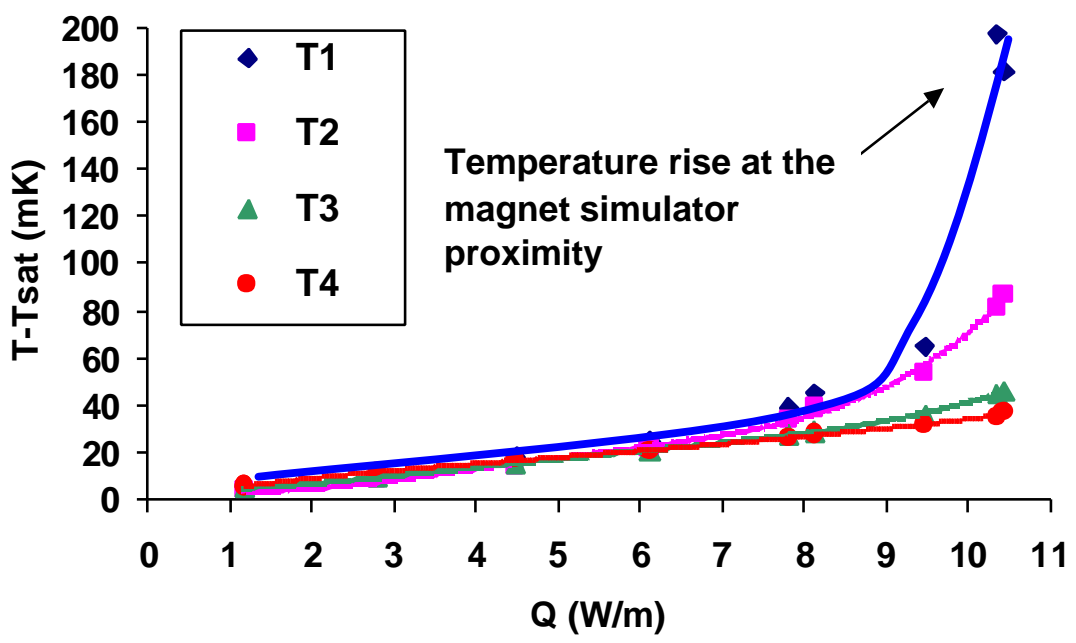

FIGURE 4. Evolution of the temperatures vs. the heat load, Tsat $=2.00 \mathrm{~K}$ 
FIGURE 4 shows the evolution of the temperature difference, for four temperature sensors distributed along the heat pattern of the last module. More than fifty heat load configurations were measured and results are consistent with this case. The results of this analysis show that, at the proximity of the heat exchanger tube, the temperature differences are proportional to the heat generated by resistive heaters. But for large heat loads, Q, the delta-T measured for sensors located in the section relative to the connecting pipes and the proximity of the heater match a quadratic fit. So, the hydraulic dimensions of the connecting pipes do not meet the requirements of the LHC high luminosity conditions. A similar behavior of hydraulic data is expected for saturated temperature lower than $2.00 \mathrm{~K}$, and heat loads higher than $8.5 \mathrm{~W} / \mathrm{m}$, but the limitation of the set-up capacity did not permit us to measure it. As a result, in order to keep a temperature margin consistent with the LHC ultimate luminosity conditions, we increased the diameter of the connecting pipes.

\section{CONCLUSIONS}

The performance of the heat exchanger tube under LHC conditions was investigated, and the LHC IR quadrupole inner triplet cooling scheme has been validated. Helium II temperatures were measured with an error of $+/-5 \mathrm{mK}$. We deduced that the wetted area of the LHC heat exchanger will be approximately $22 \%$. The temperature rise in the pressurized superfluid above saturation temperature at nominal LHC luminosity conditions will not exceed $50 \mathrm{mK}$. Nevertheless, the connecting pipe diameter has been increased in order to reduce the temperature rise resulting from the potential LHC ultimate luminosity condition.

\section{ACKNOWLEDGEMENTS}

This work was supported by the US Department of Energy. We would like to thank the LHC/ACR, LHC/ECR, LHC/CRI groups and in particular the support of R. LosserandMadoux, A. Bezaguet, E. Fernandez, C. Masure, B. Vullierme, L. Herblin, S. Pelletier, JB Bart, B. Beauquis, D. Tobias, M. Gautier, C. Berthelier and R. Vuillermet.

\section{REFERENCES}

1. Chorowski, M., Erdt, W., Lebrun, P., Riddone, G., Serio, L., Tavian, L., Wagner, U., Van Weelderen, R.,"A Simplified Cryogenic Distribution Scheme for the Large Hadron Collider," in Adv. Cryog. Eng., 43A, Plenum, New York, 1998, p.395.

2. Huang, Y., Kerby, J., Nicol, T. and Peterson, T., "Cryogenic System and Cryostat Design for the LHC IR Quadrupole Magnets," in Adv. Cryog. Eng., 43A, Plenum, New York, 1998, p.403.

3. Darve, C., Huang, Y., Kerby, J., Nicol, T. and Peterson, T., “A He II Heat Exchanger Test Unit Designed for the LHC Interaction Region Magnets," in Adv. Cryog. Eng., 45B, Plenum, 1999, p.977.

4. Blanco, E., Casas-Cubillos, J., de Prada Moraga, C. and Cristea, S., "Nonlinear Advanced Control of the LHC InnerTriplet Heat Exchanger Test Unit," presented at this conference.

5. Bon Mardion, G., Claudet, G. and P. Seyfert, "Practical Data on Steady State Heat Transport in Superfluid Helium at Atmospheric Pressure," in Cryogenics, January 1979, pp. 45-47.

6. Van Sciver, S.W., Helium Cryogenics, edited by Plenum Press, New York, 1986.

7. Darve, C., Huang, Y., Kerby, J., Nicol, T. and Peterson, T. "Experimental Investigations of He II Heat Transfer through a Short Section of LHC inner triplet Quadrupole Heat Exchanger," IEEE Trans Appl. Supercond., 2000; Vol. 11, p.1629.

8. Darve, C., Casas, J., "Measurements of temperature on LHC thermal models," Cryogenics, 2001, Vol 41 , issues 5-6, p. 319-326. 